\title{
A new fourth order embedded RKAHeM(4,4) method with error control on single layer/raster cellular neural network
}

\author{
R. Ponalagusamy - S. Senthilkumar
}

Published online: 21 July 2009

(C) Springer-Verlag London Limited 2009

Erratum to: SIViP (2009) 3:1-11

DOI 10.1007/s11760-007-0041-4

Following corrections are in order for the above mentioned paper:

In Abstract, page no. 1, left column, line no. 9: Multilayer should be read as Single layer.

In Abstract, page no. 1, left column, line no. 17, 18: RK-Embedded Centroidal Mean and RK-Embedded ContraHarmonic Mean should be ignored.

In Keywords, page no. 1, left column, line no. 21: Mulitlayer Raster, should be read as Single layer/Raster.

In page no. 2, left column, line no. 2: Mulitlayer Raster, should be read as Single layer/Raster.

In page no. 2 , right column, line no. 8, 9: RK-Embedded Centroidal Mean and RK-Embedded Contra-Harmonic Mean should be ignored.

In page no. 5, left column, Eq. 13 should be read as:

$$
\begin{aligned}
& \left|L T E_{A M}-L T E_{H e M}\right|=\frac{h^{5}}{1658880}\left[33583 f f_{y}^{4}\right. \\
& \left.\quad+40662 f^{2} f_{y}^{2} f_{y y}+33120 f^{3} f_{y y}^{2}+14444 f^{3} f_{y} f_{y y y}\right]
\end{aligned}
$$

In page no. 5, right column, Eq. 16 should be read as:

$L T E_{A M}-L T E_{H e M} \leq\left(\frac{121809}{1658880}\right) P^{4} \cdot Q h^{5}$

The online version of the original article can be found under doi:10.1007/s11760-007-0041-4.

R. Ponalagusamy $\cdot$ S. Senthilkumar $(\varangle)$

Department of Mathematics, National Institute of Technology,

Tiruchirappalli, 620015 Tamil Nadu, India

e-mail: ssenthilkumar1974@yahoo.co.in

R. Ponalagusamy

e-mail: rpalagu@nitt.edu
In page no. 5, right column, Eq. 17 should be read as:

$$
\text { Hence, }\left|y_{n+1}^{A M}-y_{n+1}^{H e M}\right| \leq \frac{121809}{1658880} P^{4} \cdot Q h^{5}
$$

In page no. 5, right column, Eq. 18 should be read as:

$$
\frac{121809}{1658880} P^{4} Q h^{5}<T O L \text { or } h<\left[\frac{13.618698 \times T O L}{P^{4} Q}\right]^{1 / 5}
$$

In page no. 6, right column, line no. 3, 4: RK-Embedded Centroidal Mean and RK-Embedded Contra-Harmonic Mean should be ignored.

In page no. 6, right column, Eq. 23 should be

ERREST $=\left|Y_{A M}-Y_{H e M}\right| \times \frac{121809}{1658880}$

In page no. 7, right column, line no. 29, Sect. 2.1, Step 2 should be read with the following sub step:

Sub Step $2.1 / *$ Calculating by RK-Embedded Heronian Mean Method*/

$$
\begin{aligned}
& k_{1}=f\left(x_{n}, y_{n}\right)=k_{1}^{*}, \quad k_{2}=f\left(x_{n}+\frac{h}{2}, y_{n}+\frac{h k_{1}}{2}\right)=k_{2}^{*}, \\
& k_{3}=f\left(x_{n}+\frac{h}{2}, y_{n}+\frac{h k_{2}}{2}\right), \quad k_{4}=f\left(x_{n}+h, y_{n}+h k_{3}\right), \\
& k_{3}=f\left(x_{n}+\frac{1}{2} h, y_{n}-\frac{1}{48} h k_{1}+\frac{25}{48} h k_{2}\right)=k_{3}^{*}, \\
& k_{4}=f\left(x_{n}+h, y_{n}-\frac{1}{24} h k_{1}+\frac{47}{600} h k_{2}+\frac{289}{300} h k_{3}\right)=k_{4}^{*}, \\
& y_{n+1}=y_{n}+h\left[\frac{k_{2}}{6}+\frac{k_{3}}{6}+\frac{2}{3}\left(\frac{k_{1} k_{2}}{k_{1}+k_{2}}\right)+\frac{2}{3}\left(\frac{k_{3} k_{4}}{k_{3}+k_{4}}\right)\right] \\
& y_{n+1}^{*}=y_{n}+\frac{h}{9}\left[k_{1}^{*}+2\left(k_{2}^{*}+k_{3}^{*}\right)+k_{4}^{*}+\sqrt{\left|k_{1}^{*}+k_{2}^{*}\right|}\right. \\
& \left.\quad+\sqrt{\left|k_{2}^{*}+k_{3}^{*}\right|}+\sqrt{\left|k_{3}^{*}+k_{4}^{*}\right|}\right]
\end{aligned}
$$


Sub Step 2.2 / Calculating by RK-Embedded Harmonic Mean Method*/

$k_{1}=f\left(x_{n}, y_{n}\right)=k_{1}^{*}, \quad k_{2}=f\left(x_{n}+\frac{h}{2}, y_{n}+\frac{h k_{1}}{2}\right)=k_{2}^{*}$,

$k_{3}=f\left(x_{n}+\frac{h}{2}, y_{n}+\frac{h k_{2}}{2}\right), \quad k_{4}=f\left(x_{n}+h, y_{n}+h k_{3}\right)$,

$k_{3}^{*}=f\left(x_{n}+\frac{h}{2}, y_{n}-\frac{h k_{1}}{8}+\frac{5 h k_{2}}{8}\right)$,

$k_{4}^{*}=f\left(x_{n}+h, y_{n}-\frac{h k_{1}}{4}+\frac{7 h k_{2}}{20}+\frac{9 h k_{3}}{10}\right)$,

$y_{n+1}=y_{n}+h\left[\frac{k_{2}}{6}+\frac{k_{3}}{6}+\frac{2}{3}\left(\frac{k_{1} k_{2}}{k_{1}+k_{2}}\right)+\frac{2}{3}\left(\frac{k_{3} k_{4}}{k_{3}+k_{4}}\right)\right]$

Fig. 2 a Original image. b After averaging and edge detection templates by employing RK-Heronian Mean Algorithm and $\mathbf{c}$ after averaging and edge detection templates by employing RK-Harmonic Mean Algorithm

Fig. 3 Maximum step-size $(\Delta T)$ yields the convergence for three different templates using two different numerical integration techniques

In page no. 8 , right column, Sub-Sect.6.1.1 should be ignored.

In page no. 9, right column, Sub-Sect.6.1.3 should be ignored.

From page no. 9 to page 10 (before Conclusion), Sect. 7 should contain as follows:

Using high power workstation, all the simulated outputs are presented below. It is observed that the actual CPU time used is equal to the simulation time. Figures $2 b$ and $c$ illustrate the results of the raster simulator obtained from a complex image of $256 * 256$ pixels. The results of the raster simulator obtained from a complex image of $256 * 256$ pixels are
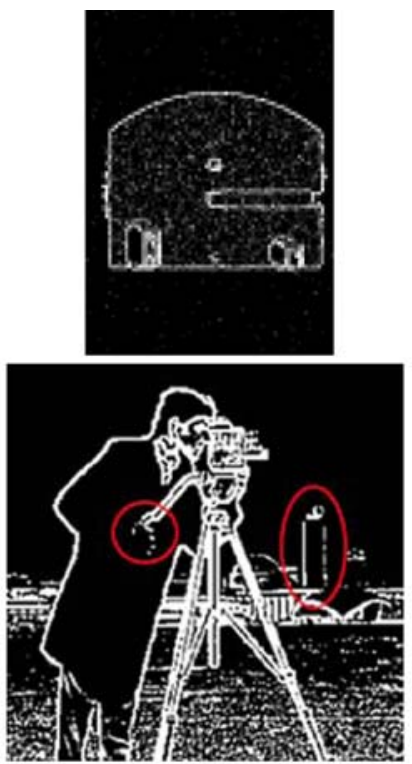

(b)
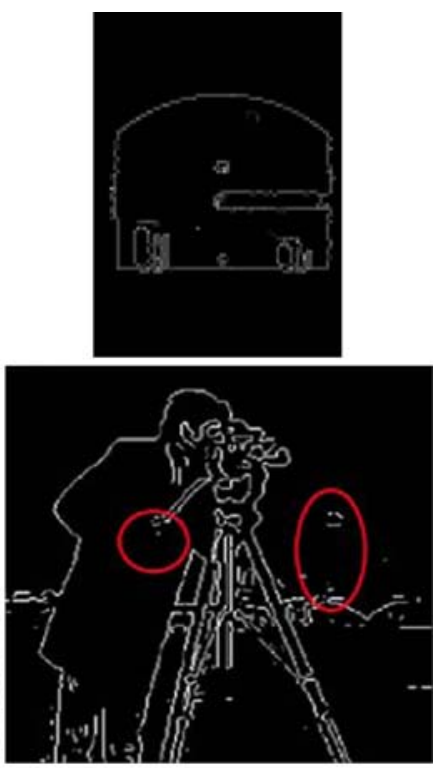

(c) (a)

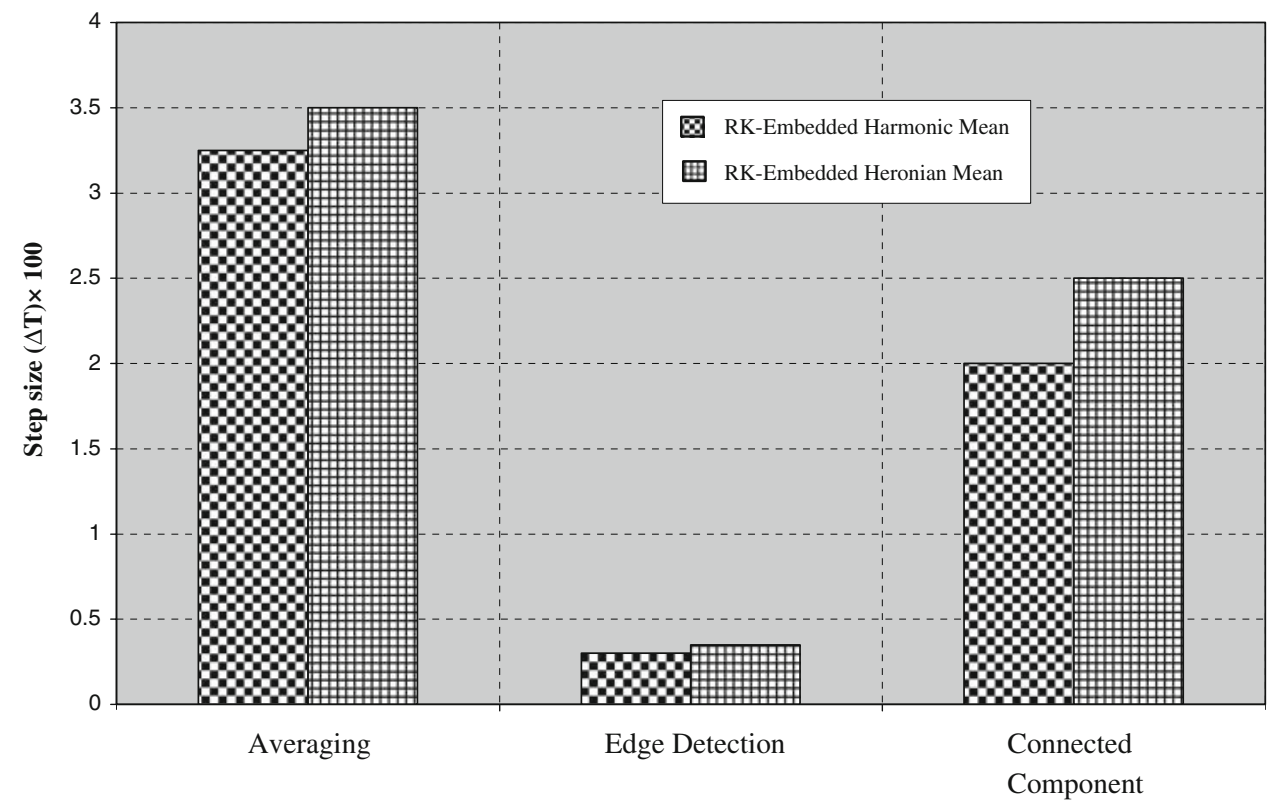


Fig. 4 Comparison of four numerical integration techniques using the edge detection template

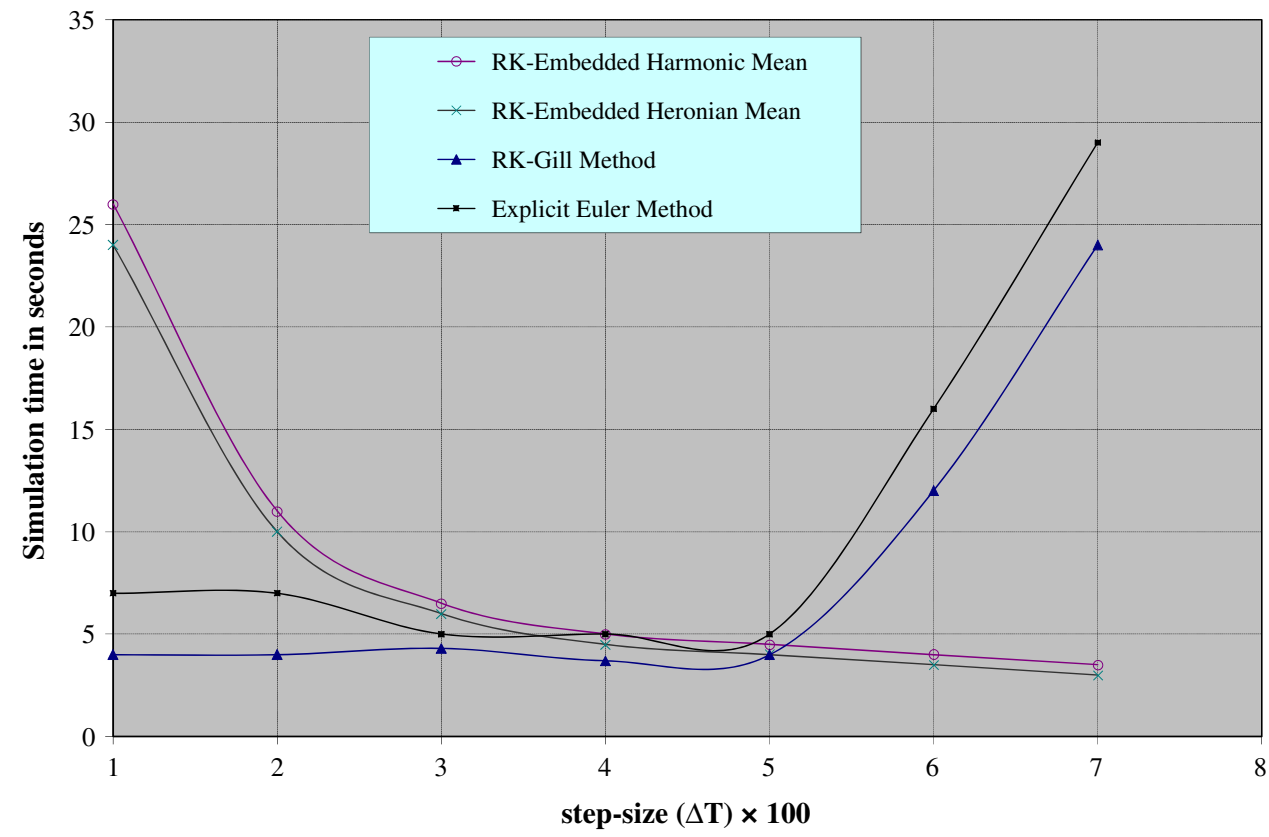

depicted respectively in figures $2 \mathrm{~b}$ and c using RK-embedded Heronian Mean and RK-embedded Harmonic Mean. On the original image in order to get the images shown in figures $2 b$ and c respectively, an averaging template followed by an edge detection template is applied. It is observed from figures $2 b$ and $c$ that the edges obtained by RK-embedded Heronian Mean is better than obtained by RK-embedded Harmonic Mean. Furthermore by raster CNN simulation the simulation took 189.56 s. It is seen from Fig. 3 that RK-embedded Heronian Mean allows us to select a maximum step-size $(\Delta \mathrm{T})$ as compared to RK-embedded Harmonic Mean method irrespective of the selection of templates.

Using edge detection template the results of Fig. 4 is obtained by simulating a small image. It is of importance to see from Fig. 4 that for a larger step-size $(\Delta T)$, RK-embedded Heronian Mean takes lesser simulation time in comparison with RK-embedded Harmonic Mean. Moreover, in specific from Fig. 4 if the step size $(\Delta \mathrm{T})$, is chosen too small, it might take many iterations, hence longer time, to achieve convergence. But, on the other hand, if the step-size $(\Delta \mathrm{T})$, taken is too large, it might not converge at all or it would converge to erroneous steady state values for the cases of adapting Explicit Euler and RK-Gill Methods. One of the notable results of the present investigation is that even for a larger time step-size $(\Delta \mathrm{T})$; the RK-embedded Heronian Mean gives better simulation results. It is seen from Table 1 that in views of Local Truncation Error, Global Truncation Error and Error Estimation the presently proposed RK-embedded Heronian Mean method is superior than the RK-embedded Harmonic Mean method.

In page no. 10, right column, Conclusion: RK-embedded Centroidal Mean and RK-Embedded Contra-Harmonic Mean should be ignored.

In page no. 11 , the corrected version of Table 1 is as follows.

Table 1 Comparison of LTE, GTE, error estimation for different fourth order four stage RK-embedded methods

\begin{tabular}{llllr}
\hline S1. no. & RK-embedded methods & Local truncation error [LTE] & Global truncation error [GTE] & Error estimation \\
\hline 1 & RK-Embedded Heronian & $L T E_{A M}-L T E_{H e M} \leq\left(\frac{121809}{1658880}\right)$ & $\left|\varepsilon_{n}\right| \leq\left(\frac{h^{4}}{1658880 L D}\right) M\left(e^{D L\left(x_{n}-x_{0}\right)}-1\right)$ & ERREST \\
& Mean (Present Paper) & $P^{4} \cdot Q h^{5}=\left|y_{n+1}^{A M}-y_{n+1}^{H e M}\right|$ & & $=\left|Y_{A M}-Y_{H e M}\right| \times \frac{121809}{1658880}$ \\
& & $\leq \frac{121809}{1658880} P^{4} \cdot Q h^{5}$ & \\
2 & $L T E_{A M}-L T E_{H M} \leq\left(\frac{5469}{69120}\right)$ & $\left|\varepsilon_{n}\right| \leq\left(\frac{h^{4}}{69120 L D}\right) M\left(e^{D L\left(x_{n}-x_{0}\right)}-1\right)$ & ERREST \\
& RK-Embedded & $P^{4} \cdot Q h^{5}=\left|y_{n+1}^{A M}-y_{n+1}^{H M}\right|$ & $=\left|Y_{A M}-Y_{H M}\right| \times \frac{5469}{69120}$ \\
\hline
\end{tabular}

